\title{
Characterizing and reducing the POL-2 instrumental polarization
}

Per Friberg, David Berry, Giorgio Savini, Dan Bintley, Jessica Dempsey, et al.

Per Friberg, David Berry, Giorgio Savini, Dan Bintley, Jessica Dempsey, Sarah Graves, Harriet Parsons, "Characterizing and reducing the POL-2 instrumental polarization

," Proc. SPIE 10708, Millimeter, Submillimeter, and Far-Infrared Detectors and Instrumentation for Astronomy IX, 107083M (9 July 2018); doi: $10.1117 / 12.2314345$

Event: SPIE Astronomical Telescopes + Instrumentation, 2018, Austin, Texas, United States 


\title{
Characterizing and reducing the POL-2 instrumental polarization
}

\author{
Per Friberg $^{\mathrm{a}}$, David Berry ${ }^{\mathrm{a}}$, Giorgio Savini ${ }^{\mathrm{b}}$, Dan Bintley ${ }^{\mathrm{a}}$, Jessica Dempsey ${ }^{\mathrm{a}}$, Sarah Graves ${ }^{\mathrm{a}}$, \\ and Harriet Parsons ${ }^{\mathrm{a}}$ \\ ${ }^{a}$ East Asia Observatory, 660 N. A'ohōkū Pl., Hilo, USA \\ ${ }^{\mathrm{b}}$ University College London, Department of Physics and Astronomy, Gower Street, London \\ WC1E 6BT, UK
}

\begin{abstract}
Sub-millimeter polarization observations using the POL-2 instrument mounted on the dual wavelength $(850 / 450$ $\mu \mathrm{m}) 10 \mathrm{k}$ pixel sub-millimeter camera SCUBA-2 is in high demand on the James Clerk Maxwell Telescope (JCMT). The high level of Instrumental Polarization (IP) generated by the Gore-Tex ${ }^{\mathrm{TM}}$ wind blind protecting the telescope is a hampering factor for these observations. The wind blind both introduces an overall linear polarization and a four lobed polarization footprint seen on strong point sources after removal of a beam averaged IP. During commissioning an IP model was developed for the $850 \mu \mathrm{m}$ band but a good $450 \mu \mathrm{m}$ model was lacking. This paper describes the effort to improve the $850 \mu \mathrm{m}$ IP model, establish a $450 \mu \mathrm{m}$ model and the work to understand and model the IP. During the work the wind blind was removed for a month to isolate the contribution of the wind blind from other sources of the IP. A theoretical model for the non wind blind generated IP has been developed. However, a theoretical model for the wind blind IP is still being worked on.
\end{abstract}
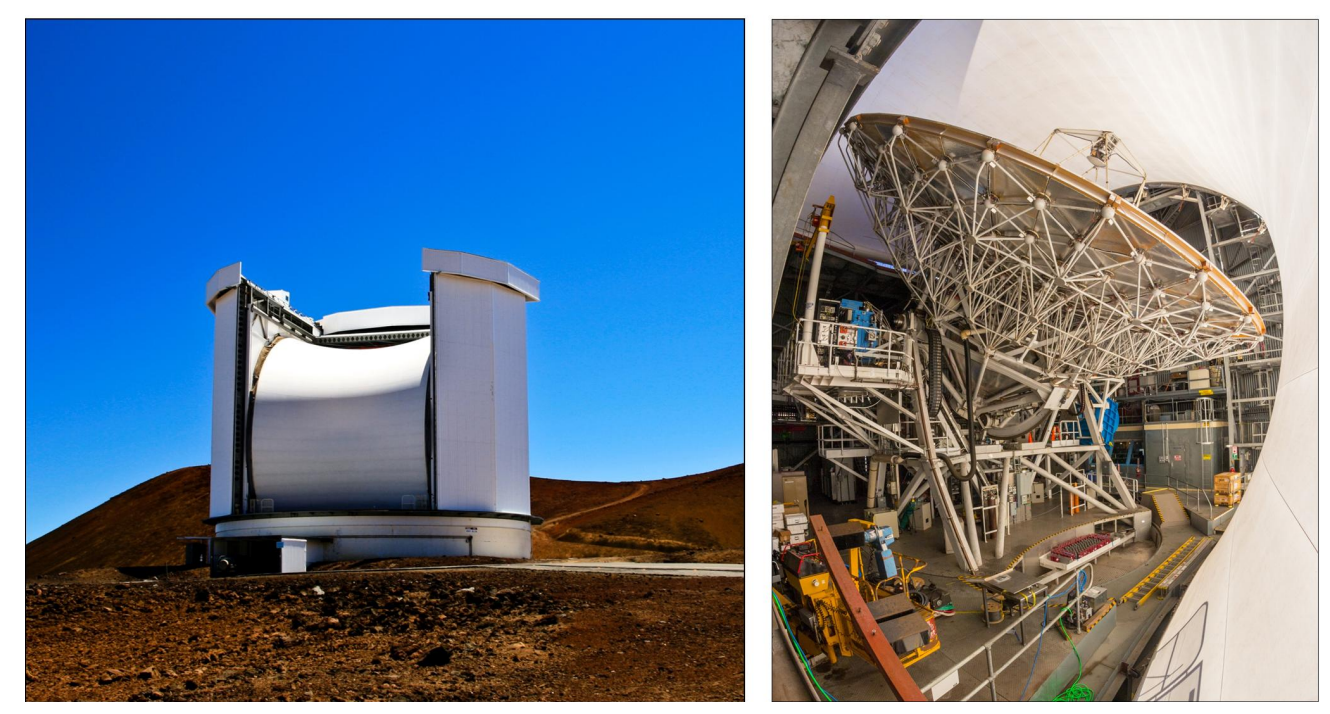

Figure 1. (left) The JCMT in its usual mode of operation with a saddle shaped protective wind blind in place (courtesy of ROE Photolabs) (right) Inside the enclosure showing the wind blind in front of the JCMT dish.

Keywords: Polarimetry, JCMT, Instrumental Polarization, Submillimeter Instrumentation, SCUBA-2, POL-2

Further author information: (Send correspondence to Per Friberg)

Per Friberg.: E-mail: p.friberg@eaobservatory.org, Telephone: +1 8089696522

Millimeter, Submillimeter, and Far-Infrared Detectors and Instrumentation for Astronomy IX, edited by Jonas Zmuidzinas, Jian-Rong Gao, Proc. of SPIE Vol. 10708, 107083M

(C) 2018 SPIE · CCC code: 0277-786X/18/\$18 · doi: 10.1117/12.2314345 


\section{INTRODUCTION}

Polarization observations are fundamental for understanding the importance of magnetic fields for cloud structure, cloud collapse and star formation. The POL-2 instrument on the SCUBA-2 camera is capable of mapping polarization over large areas of the sky in a reasonable time. This makes it possible to map the large scale distribution of magnetic fields and investigate its importance for cloud development. A large survey (BISTRO) and many smaller programs are using this capacity at $850 \mu \mathrm{m}$, complementing high angular resolution observations done by interferometers such as ALMA. Polarization observations at $450 \mu \mathrm{m}$ have so far been very limited due to the need for very good weather and the lack of a good IP model at this wavelength.

In order to get the true sky polarization, the polarization introduced by the telescope and instrument first has to be removed. An IP model is applied to a total intensity map and the resulting polarization is removed from the polarization map of the region. Any uncertainty in the IP will directly affect the polarization map produced. Thus, it is critical to characterize and understand the IP as well as possible. In the case of the JCMT, the IP is dominated by the Gore-Tex ${ }^{\mathrm{TM}}$ wind blind protecting the telescope from wind, solar radiation, precipitation and dust. An obvious method to reduce the IP would be to remove the wind blind. However, this has implications for the telescope performance and operation. The maximum wind speed for safe operation is almost halved. There are effects on pointing due to wind buffering and surface accuracy due to wind pressure. Further, looking anywhere in the vicinity of the Sun would be a mortal danger to the telescope, severely limiting daytime observations. The wind blind is very effective in reducing the radiation from the Sun - in fact the JCMT routinely uses the Sun as an alignment source. Comet and other solar system observations are often performed in the daytime. A month of operation without the wind blind has shown clearly that routine operation without the wind blind is not a realistic option. The loss in observing time is substantial and the telescope efficiency is reduced due pointing jitter and surface degradation.

\section{POL-2 AND SCUBA-2}

SCUBA-2 is a bolometric camera with 5120 bolometers in each of the $850 \mu \mathrm{m}$ and $450 \mu \mathrm{m}$ focal planes. Both wavelengths are always observed together but the noise level at $450 \mu \mathrm{m}$ is very weather dependent, limiting the useful observing time at this wavelength. Observing is done by scanning the telescope repeatably over the source region for the duration of an observation. The extensive cross linking in the data generated by this scan pattern allows the data reduction to separate common mode signals due to the atmosphere and instrument, as well as $1 / \mathrm{f}$ noise from the astronomical signal. A description of SCUBA-2, its observing modes and data reduction can be found in. ${ }^{1-3}$ POL- $2^{4}$ is an auxiliary instrument that is inserted in front of SCUBA-2 to observe polarization. It consists of a rotating achromatic $\lambda / 2$ plate and an analyzer grid. The spinning wave plate and analyzer modulates linear polarization and the modulation, as well as the total intensity, is detected by SCUBA2. In POL-2 observations the wave plate spins fast enough to generate raw $\mathrm{Q}, \mathrm{U}$ and $\mathrm{I}$ values at each position before the telescope has moved significantly. A modified version of the SCUBA-2 data reduction then generates astronomical Q, U and I maps from the raw data by removing signals not correlated with the astronomical background. This includes removal of the polarized background generated by atmospheric emission polarized by the telescope optics and wind blind. SCUBA-2 and POL-2 are mounted on the left Nasmyth platform on the JCMT. Hence, any IP generated by the wind blind or optics that moves with the telescope will rotate with respect to the instrument.

\section{DESCRIPTION OF THE WIND BLIND}

The Gore-Tex ${ }^{\mathrm{TM}}$ wind blind is made of a fabric consisting of a basket weave using threads of size $\sim 250 \mathrm{x} 80 \mu \mathrm{m}$. The threads are made of thin Gore-Tex ${ }^{\mathrm{TM}}$ strips triple folded. However, two threads are used together in making the basket weave such that the weave has an apparent spacing of $\sim 500 \mu \mathrm{m}$, see figure 2 . The Gore-Tex ${ }^{\mathrm{TM}}$ fabric is covered by a Gore-Tex ${ }^{\mathrm{TM}}$ film making the wind blind impermeable to water and wind. The wind blind is made by sewing together 1.2 meter wide strips of the Gore-Tex ${ }^{\mathrm{TM}}$ material oriented horizontally across the aperture. Hence, the warp is horizontal and the weft vertical. The material is $\sim 0.3 \mathrm{~mm}$ thick apart from in the seams. In the seams, the material is four-folded but the relative area is very small. The strips were cut about $10 \%$ narrower in the middle to generate the saddle shape. Thus the threads in the weave are roughly oriented 


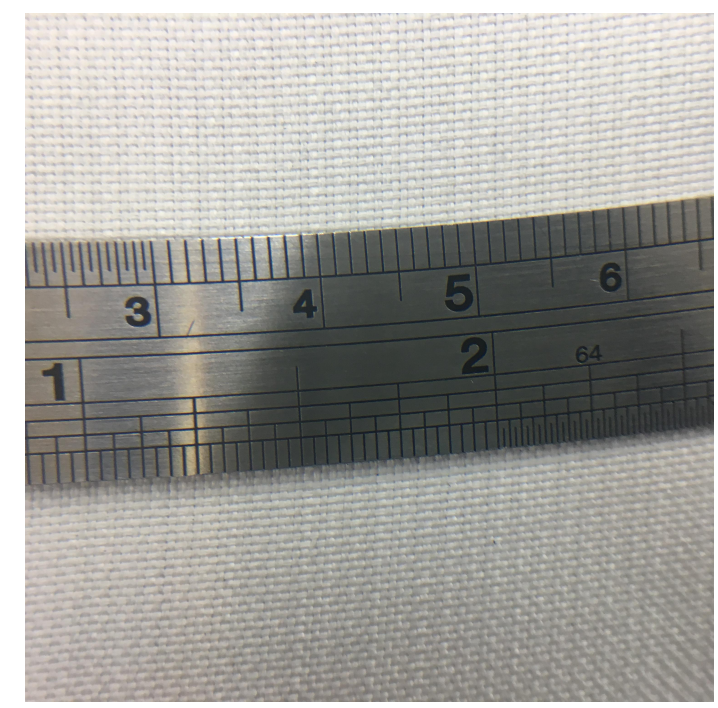

Figure 2. Close up image of a spare sample of wind blind material. Some optical optical distortion is apparent from the curvature of the ruler. The top scale on the ruler is in $\mathrm{mm}$, bottom inches. As two threads being used together, the apparent thread pattern is $\sim 500 \mathrm{um}$ instead of $\sim 250 \mu \mathrm{m}$. Counting threads, the weave appears asymmetric in thread count of about $2 \%$ in the two directions.

vertically/horizontally across the full aperture - the shape is not generated by tensioning and stretching of the material. However, to inhibit motions induced by the wind, the wind blind is in fact tensioned, but without the material being noticeable stretched.

Gore-Tex ${ }^{\mathrm{TM}}$ is Polytetrafluoroethylene (PTFE or Teflon ${ }^{\mathrm{TM}}$ ) which is stretched during the fabrication, creating a material with micro pores. Due to the stretching, the density and dielectric constant is lower than for bulk PTFE. During the testing of the wind blind material in the 1980's, the index of refraction was measured to be 1.25 compared to the value of 1.44 for bulk PTFE. The low index of refraction reduces reflection losses and combined with low absorption coefficient and high strength it makes Gore-Tex ${ }^{\mathrm{TM}}$ an excellent material for a wind blind. This information about the wind blind material is obtained from JCMT design memos. ${ }^{5-7}$

\section{MEASURED IP}

The IP has been measured using Uranus as a source which is assumed to be effectively unpolarized. Uranus is smaller than the telescope half power beam width; $\sim 13^{\prime \prime}$ and $\sim 7^{\prime \prime}$ at $850 \mu \mathrm{m}$ and $450 \mu \mathrm{m}$, respectively. Hence, any symmetric polarization will average out within the resolution. Further, during the period of the observations, the position of Uranus allowed observations to be taken over a large elevation range. This is important since the IP has elevation dependence. Alternative sources like Mars are larger, making resolution of polarized limb polarization an issue. Further, the elevation range on Mars would have been limited albeit the signal to noise ratio would have been better. To achieve a useful signal to noise ratio at $450 \mu \mathrm{m}$ very good weather is required. This is one of the main reason it has been troublesome to measure the IP at $450 \mu \mathrm{m}$. A large number of 5 minutes maps were obtained and reduced in a consistent manner to generate the IP data. To generate the IP data the Q, U and I flux has been integrated over a $40^{\prime \prime}$ aperture. The average I value was used to generate $\mathrm{q}$ $(\mathrm{Q} / \mathrm{I})$ and $\mathrm{u}(\mathrm{U} / \mathrm{I})$ values for the plots below.

\subsection{Without the wind blind}

The measured $\mathrm{q}$ and $\mathrm{u}$ as observed without the wind blind are shown in figure 3 together with the best fit to the data. Only the $850 \mu \mathrm{m}$ data has been fitted. The fit displayed on the $450 \mu \mathrm{m}$ plot is a scaled version of the 850 $\mu \mathrm{m}$ fit - the scaling being proportional to the square root of the wavelength ratio. While a similar fit can be done to the $450 \mu \mathrm{m}$ the result has large error bars. Instead, we show a scaled version of the $850 \mu \mathrm{m}$ IP fit is, within 
the errors, consistent with the $450 \mu \mathrm{m}$ data. As described in section 5.1 the IP without the wind blind can be well modeled by polarization from the six relay mirrors between the telescope and the POL-2 instrument.
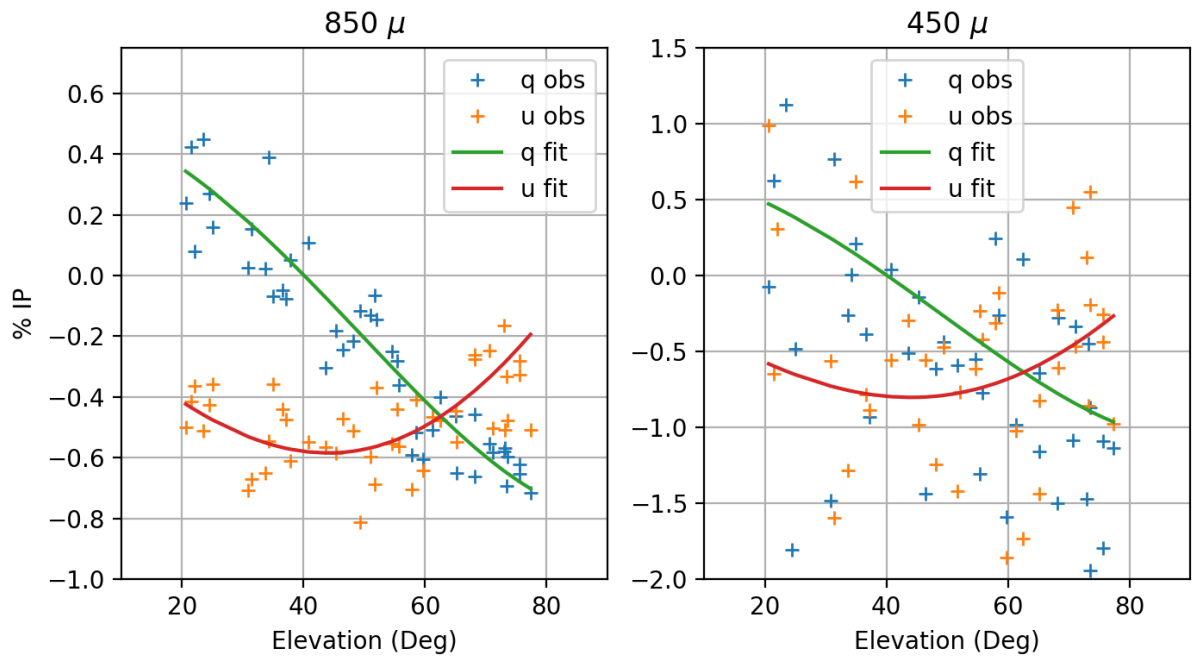

Figure 3. Measured IP and the best fitted model without the wind blind in place. Left : $\mathrm{q}$ and $\mathrm{u}$ data for $850 \mu \mathrm{m}$ and the fit to the $850 \mu \mathrm{m}$ data. Right: $\mathrm{q}$ and $\mathrm{u}$ data for $450 \mu \mathrm{m}$ and a scaled version of the $850 \mu \mathrm{m}$ fit. Note that the intensity scale for the $450 \mu \mathrm{m}$ plot is not the same as for the $850 \mu \mathrm{m}$ plot.

\subsection{With the wind blind}

The observed IP with the wind blind in place is shown in figure 4. While the IP without the wind blind has a similar shape at $850 \mu \mathrm{m}$ and $450 \mathrm{\mu m}$, this is not the case with the wind blind in place. The measured $\mathrm{q}$ and $\mathrm{u}$ shows a rotation of about 90 degrees between $850 \mu \mathrm{m}$ and $450 \mu \mathrm{m}$. The IP is dominated by the wind blind - the IP is approximately $2 \%$ with and $0.6 \%$ without the wind blind.
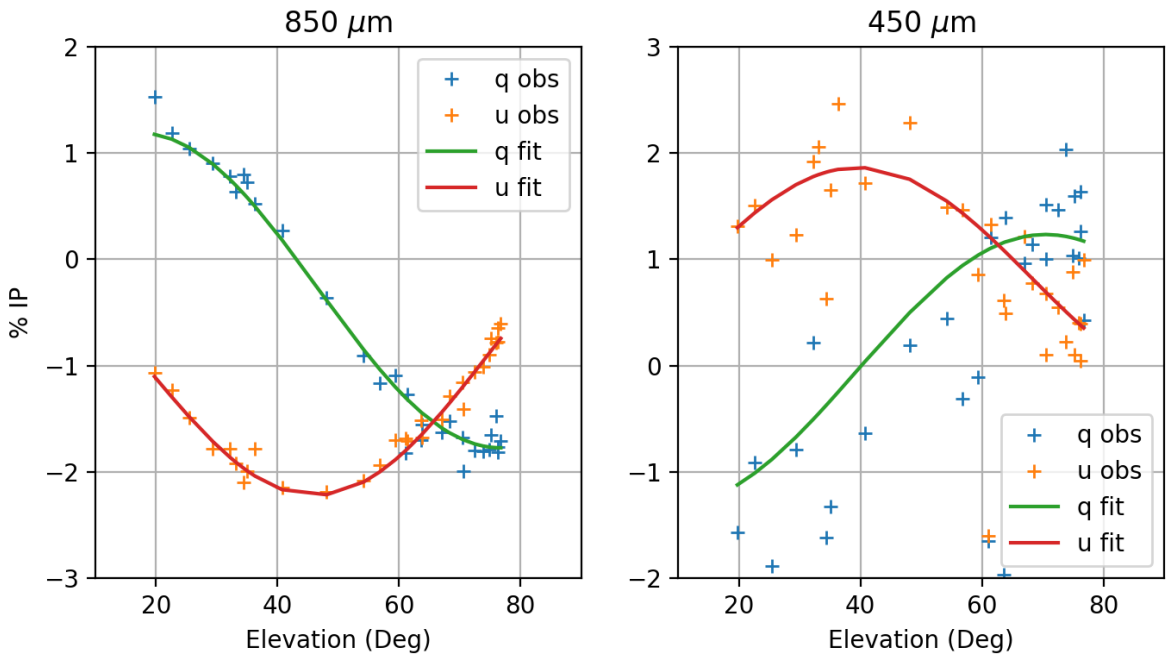

Figure 4. Measured IP with the wind blind in place. Left: Normalized Q and U data for $850 \mu \mathrm{m}$ and the fit to the 850 $\mu \mathrm{m}$ data. Right: Normalized $\mathrm{Q}$ and $\mathrm{U}$ data for $450 \mu \mathrm{m}$ and the fit to the $450 \mu \mathrm{m}$ data. The IP is rotated about 90 degrees between the 850 and $450 \mu \mathrm{m}$ focal planes. 


\subsection{The IP shape with the wind blind}

The IP is not constant across the point spread function of the telescope. While cross polarization is well known in side lobes, this is a features in the main lobe. The observed Q and U images are shown in the left panels of figure 5. Even though Uranus is much smaller than the beam size and unresolved, the apparent shape is clearly not symmetric. The right panels contain the $\mathrm{Q}$ and $\mathrm{U}$ images after the IP has been removed by using a constant percentage of the total intensity image (I). The integrated polarization is now close to zero as expected but there is a residual pattern. The $\mathrm{Q}$ and $\mathrm{U}$ maps are generated by subtracting the polarization in two orthogonal directions. If the beam patterns in the two directions are different, the IP will not be constant across the beam. The effect seen can be generated by having slightly different beam sizes in the two polarization directions. This would imply that the wind blind not only polarizes the incoming radiation but, it also alters the illumination of the dish depending on the polarization.
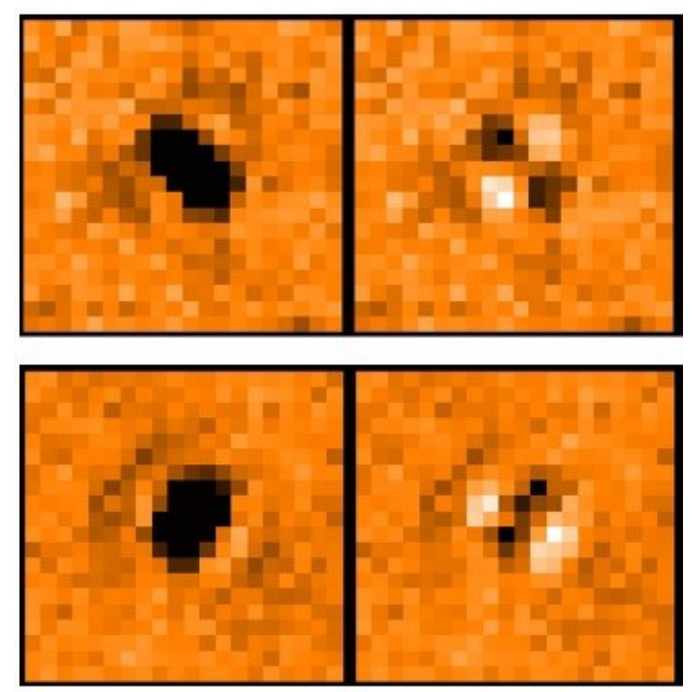

Figure 5. IP footprint in maps of Uranus. Left column Q (top) and U (bottom) is the maps uncorrected for IP observed on Uranus. The image is clearly not circular. Right column the same maps with a IP removed based on a constant fraction of the total intensity map. The IP correction based on the total intensity removes the average IP but leaves a residual footprint. The map is taken at an elevation of about 60 degrees making both $q$ and $u$ negative (black). The IP rotates with elevation and becomes horizontal at lower elevations but also less elongated implying the illumination differences are smaller at low elevations.

\section{MODELING}

Understanding how the IP is generated would be beneficial for improving our $450 \mathrm{\mu m}$ IP models. The IP data at $450 \mu \mathrm{m}$ is limited and noisy due to the weather sensitivity. Fitting a known shape would improve the quality of the fit. The data conclusively shows that wind blind is the main source of the IP, and since it is unlikely we can operate without the wind blind for any extended periods we need to work on improving the IP models instead of removing the cause of the IP.

\subsection{Without the wind blind}

The IP without the wind blind is smaller and similar at $850 \mu \mathrm{m}$ and $450 \mu \mathrm{m}$. We have considered two main sources: i) contributions from the instrument itself, and ii) contributions from the six relay mirrors between the Cassegrain focus and POL-2. An instrumental contribution has been predicted but is likely small. Any IP generated by the instrument will not rotate with the elevation of the telescope. We have looked for IP components not affected by the elevation and not found any above the noise limit.

Of the six relay mirrors between the Cassegrain focus and POL-2, four move with the telescope and rotate with respect of instrument. Two of the mirrors are on the Nasmyth and are fixed relative to the instrument. 
The mirrors generate polarization since the reflection coefficients, while close to one, are slightly different for polarization aligned with and perpendicular to the plane of incidence - the Transverse Magnetic (TM) and the Transverse Electric (TE) case, respectively. This effect is easily calculated, and while the effect from each mirror is small $(\sim 0.1 \%)$, the overall contribution is detectable. All the mirrors line up (share the same plane of incidence) when the telescope points to zenith. When pointing close to the horizon, the two Nasmyth mirrors are rotated 90 degrees with respect to the other four mirrors. Thus, we would expect the maximum contribution towards zenith to decrease towards lower elevation. The contribution the mirrors make increases with the angle of incidence. The mirrors with largest angle of incidence are all fixed relative the telescope so the cancellation is limited. Thus, we would expect to see the largest IP towards zenith decreasing slightly with elevation. The measured IP without the wind blind is compared with the mirror model in Figure 6. The only fitted parameter in the model is the DC resistivity of the Aluminum Alloy used in the mirrors - the model shown uses 2.8 times the DC resistivity expected from the Aluminum Alloy. Such a discrepancy is not unusual - it is common to observe higher resistivity for reflections. ${ }^{8}$ The reflection and polarization is expected to scale with the square root of the frequency - this has been used in figure 3 to scale the $850 \mu \mathrm{m}$ fit in the $450 \mu \mathrm{m}$ plot.
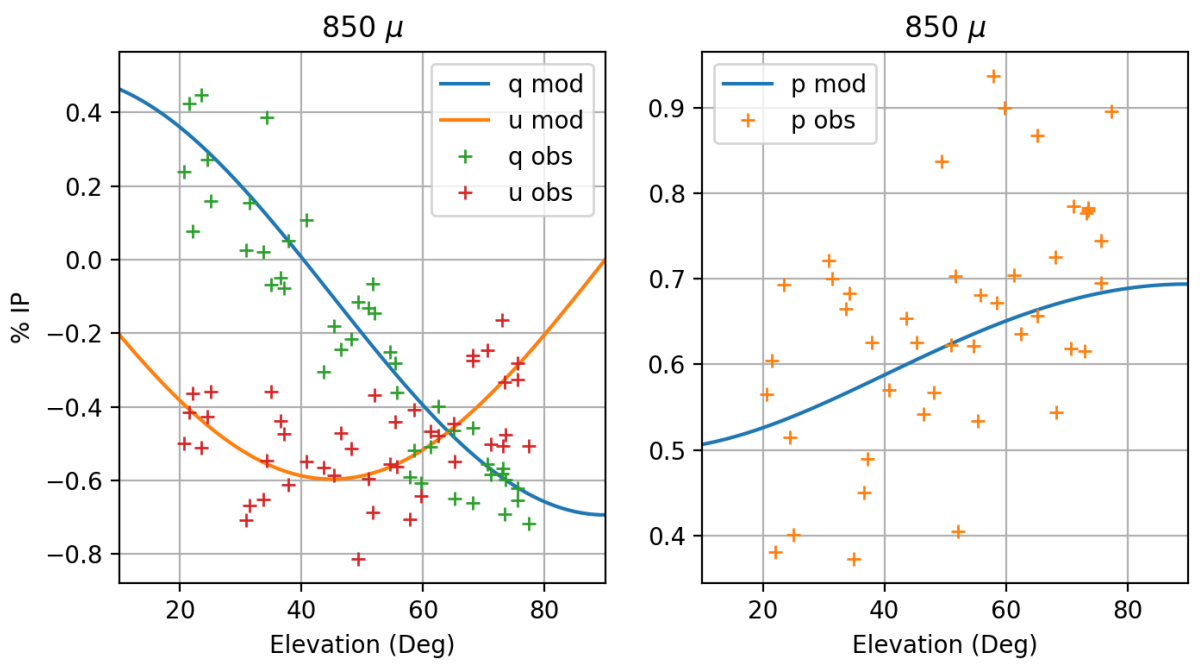

Figure 6. Comparison between the data and the model for IP generated by the six relay mirrors without the wind blind. (right) Observed (obs) and theoretical model (mod) for q and $u$. (right) Observed (obs) and theoretical model (obs) for degree of polarization. As predicted the degree of polarization is highest when the telescope points to zenith and decreases towards the horizon.

\subsection{With the wind blind}

The wind blind dominates the IP and behaves differently at $850 \mu \mathrm{m}$ and $450 \mu \mathrm{m}$. At $850 \mu \mathrm{m}$ the IP generated by the wind blind is aligned to that of the relay mirrors, while at $450 \mu \mathrm{m}$ the wind blind IP is rotated by 90 degrees with respect to the IP from the relay mirrors. Simple estimates for the IP generated by the wind blind by itself after the relay mirror's contribution has been removed give $1.5 \%$ and $2.5 \%$ at $850 \mu \mathrm{m}$ and $450 \mu \mathrm{m}$ respectively. Known dependency of achromatic wave plates? fast axis with wavelength can be ruled out as the cause of the difference between the $850 \mu \mathrm{m}$ and $450 \mu \mathrm{m}$ IP. The value for the offset between $850 \mu \mathrm{m}$ and $450 \mu \mathrm{m}$ has been measured to less than 2 degrees for the POL- $2 \lambda / 2$ wave plate?

One possible way for the wind blind to generate the IP is by reflection and absorption. The wind blind is a dielectric film, which due to the curvature, reflects vertical and horizontal polarization differently. The wind blind curves much more in the vertical direction than in the horizontal direction. As a result the Brewster angle is approached in the vertical direction for vertical polarization (TM). Thus the dish illumination is more uniform in the vertical direction for vertical polarization than for horizontal polarization. The effect of a dielectric film can be modeled and gives results that are qualitatively but not quantitatively similar to what is observed at 
$850 \mu \mathrm{m}$. As a result of the difference in vertical illumination between vertical and horizontal polarization an IP footprint (see figure 5), is predicted similar to what is observed. However, the predicted effect is only 0.2 $0.3 \%$ compared to the observed level of $1.5 \%$ at $850 \mu \mathrm{m}$. In addition no 90 degrees rotation of the $450 \mu \mathrm{m}$ is predicted. A curved dielectric film would need to have a index of refraction as high or higher than bulk PTFE (1.44) or be much thicker to produce the magnitude of the observed IP at $850 \mu \mathrm{m}$. The dielectric film model can not explain the observed $450 \mu \mathrm{m}$ IP, which is rotated by 90 degrees compared to the $850 \mu \mathrm{m}$ IP. Thus the effects of a dielectric film are not enough to explain the IP from the wind blind.

The dielectric film model does not include scattering by the threads or asymmetries in the weave as sources for polarization. Taking these factors into account is more complicated, but is the next step. In order to model this we need to get a better description of the variation in the weave. The dielectric film model does give some insights into what is needed to get the observed IP. From the observations of the footprint at $850 \mathrm{\mu m}$ we need to have a polarization dependent dish illumination. Thus, a simple model with the weave uniformly more transparent for one polarization than the other across the aperture is not consistent with the observations. Further, such an asymmetry could not easily explain the difference between $850 \mu \mathrm{m}$ and $450 \mu \mathrm{m}$. For scattering to explain the observations it needs to be non uniform across the aperture and strongly differeing between $850 \mu \mathrm{m}$ and $450 \mu \mathrm{m}$. Samples of the wind blind material were tested before the construction and showed losses of order a few $\%$ at $850 \mu \mathrm{m}$ while the losses at $450 \mu \mathrm{m}$ were up to $15 \%$. Particular, at $450 \mu \mathrm{m}$, this is higher than predicted from reflection and absorptive losses in a dielectric film. This is not surprising considering the weave has features of order of the wavelength at $450 \mu \mathrm{m}$. Due to the curvature of the wind blind, the projected structure in the wind blind will have shorter spacing away from the aperture center, particularly in the vertical direction where the curvature is larger.
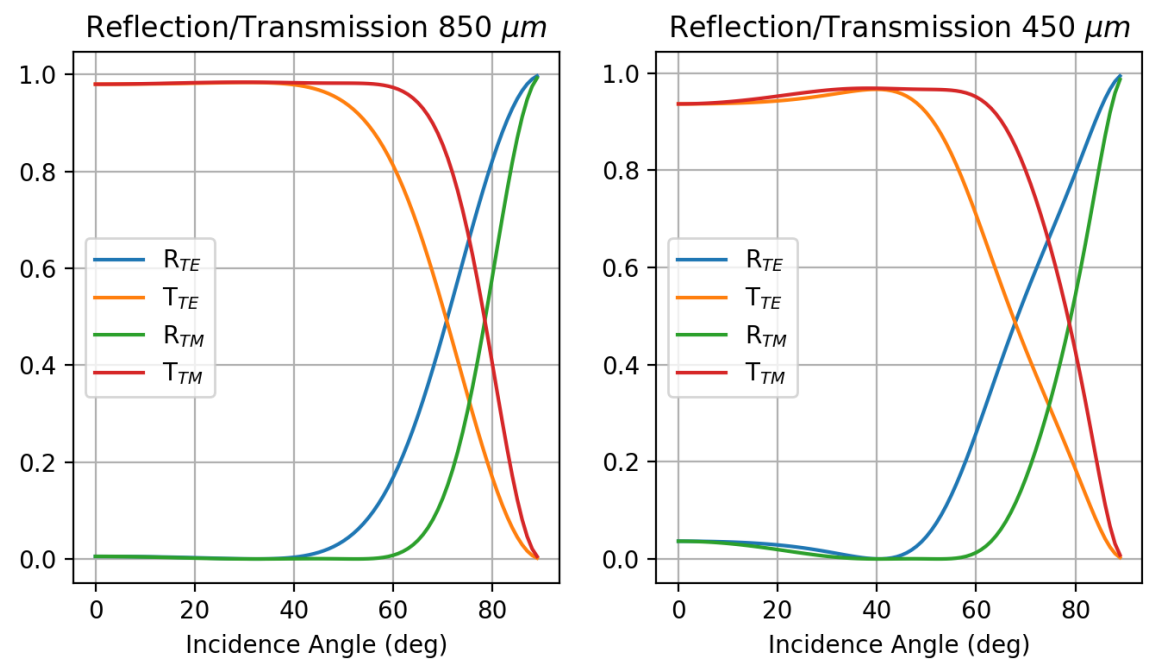

Figure 7. Computed power reflection and transmission for TE and TM waves as function of incidence angle at $850 \mu \mathrm{m}$ and $450 \mathrm{\mu m}$. In the horizontal direction the wind blind angle of incidence is about 20 degrees at the edge of the aperture. In the vertical direction the angle of incidence is about 50 degrees at the edge. Thus, the transmission is only markedly different between the two polarizations at the dish edge in the vertical direction. The effect on the IP is small due to the small area of the dish affected. Reflection causes a $6 \%$ loss for low angles of incidence at $450 \mu \mathrm{m}$. For increasing angle of incidence this transmission first increases due to positive interference close to $\lambda / 2$ before decreasing again.

\section{SUMMARY}

We have improved the empirically measured IP with POL-2 at $850 \mu \mathrm{m}$ and generated a model at $450 \mu \mathrm{m}$. This has been done with and without the wind blind in place. The results without the wind blind can be modeled by polarization generated by the relay mirrors. The IP with the wind blind is more pronounced and different for $850 \mu \mathrm{m}$ and $450 \mu \mathrm{m}$. This difference is not yet understood and more work is ongoing to model asymmetries 


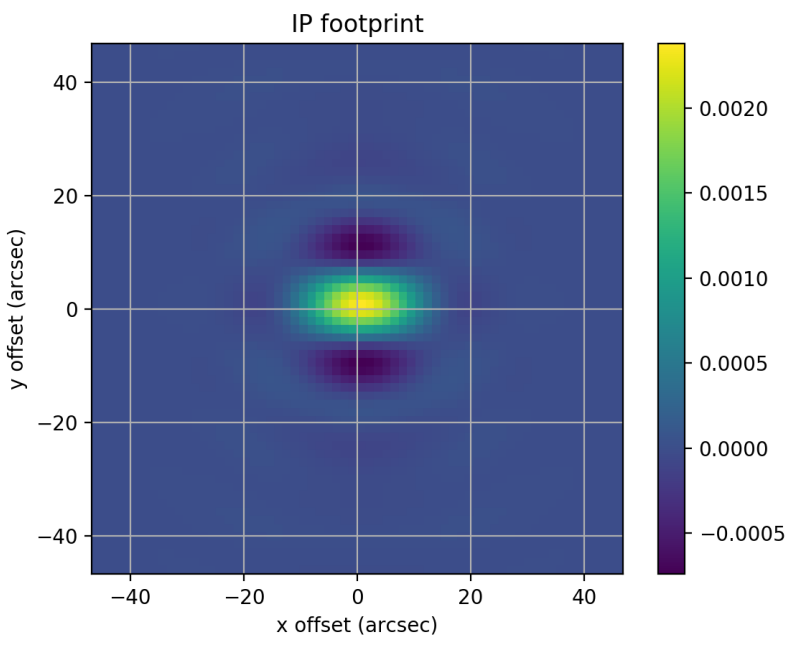

Figure 8. The $850 \mu \mathrm{m}$ IP footprint as calculated from assuming the wind blind is a dielectric film reflecting TE and TM polarization slightly differently. The main effect is that, due to the curvature of the wind blind, vertical polarization is reflected less with vertical offset from the center of the dish. The difference in illumination generates an overall vertical polarization. The telescope beam will also be slightly different for vertical and horizontal polarization, generating an IP footprint. This should be compared to figure 5. The difference in rotation is due to the calculation not includes any rotation with elevation. Such rotation is present in the observed IP footprint due to instrument location at the Nasmyth.

in and scattering from the wind blind. Higher signal to noise $450 \mu \mathrm{m}$ data and measurement of the $450 \mu \mathrm{m}$ IP footprint would be helpful for this modeling.

\section{ACKNOWLEDGMENTS}

For the period 1987 until February 2015 the JCMT was operated by the Joint Astronomy Centre on behalf of the UK Science and Technologies Facilities Council (STFC), the Netherlands Organisation for Pure Research, and the National Research Council of Canada. From March 2015 the telescope has been operated by the East Asian Observatory on behalf of the National Astronomical Observatory of Japan, the Academia Sinica Institute of Astronomy and Astrophysics of Taiwan, the Korea Astronomy and Space Science Institute, the National Astronomica Observatories of China and the Chinese Academy of Sciences. Additional operational support funding is also provided by STFC and participating universities in the UK and Canada.

\section{REFERENCES}

[1] Dempsey, J. T., Friberg, P., Jenness, T., Tilanus, R. P. J., Thomas, H. S., Holland, W. S., Bintley, D., Berry, D. S., Chapin, E. L., Chrysostomou, A., Davis, G. R., Gibb, A. G., Parsons, H., and Robson, E. I., "SCUBA-2: on-sky calibration using submillimetre standard sources," MNRAS 430, 2534-2544 (2013).

[2] Holland, W., Bintley, D., Chapin, E. L., Chrysostomou, A., Davis, G. R., Dempsey, J. T., Duncan, W. D., Fich, M., Friberg, P., Halpern, M., and et.al., "SCUBA-2: the 10000 pixel bolometer camera on the James Clerk Maxwell Telescope," MNRAS 430, 2545-2573 (2013).

[3] Chapin, E. L., Berry, D. S., Gibb, A. G., Jenness, T., Scott, D., Tilanus, R. P. J., Economou, F., and Holland, W., "SCUBA-2: iterative map-making with the sub-millimetre user reduction facility," MNRAS 430, 2513$2533(2013)$.

[4] Friberg, P., Bastien, P., Berry, D. S., Savini, G., Graves, S., and Pattle, K., "POL-2: a polarimeter for the James-Clerk-Maxwell Telescope," in [Millimeter, Submillimeter, and Far-Infrared Detectors and Instrumentation for Astronomy VIII], Holland, W. S. and Zimudas, J., eds., Proc. SPIE 9914, doi: 10.111712.2231943 (2016). 
[5] Street, R. L. T., "Membrane Material Selection." JCMT design memo ASR/MT/T/426/RLTS (1983).

[6] Hall, J. E. and Street, R. L. T., "REVIEW OF THE CONCEPTUAL DESIGN OF THE MT MEMBRANE." JCMT design memo ASR/MT/T/601/JEH/RLTS (1984).

[7] Lamb, J., "ELECTROMAGNETIC DESGIN OF UK/NL MILLIMETREWAVE TELESCOPE EFFECTS OF MEMBRANE ON GAIN AND POLARIZATION." JCMT design memo ASR/MT/T/691/JL (1984).

[8] Renbarger, T., Dotson, J. L., and Novak, G., "Measurements of submillimeter polarization induced by oblique reflection from aluminum alloy," Applied Optics 37, 6643-6647 (1998).

[9] Pancharatnam, S., "Achromatic combinations of birefringent plates part ii. an achromatic quarter-wave plate," Proceedings of the Indian Academy of Sciences - Section A 41, 137-144 (1955).

[10] Savini, G., Ade, P. A. R., House, J., Pisano, G., Haynes, V., and Bastien, P., "Recovering the frequency dependent modulation function of the achromatic half-wave plate for pol-2: the scuba-2 polarimeter," Applied Optics 48, 2006-2013 (2009). 